\title{
Validation of the Digital Correlation ECE Measurement Technique using Low-Frequency Fluctuation in LHD*)
}

\author{
Hayato TSUCHIYA, Shigeru INAGAKI ${ }^{1)}$, Tokihiko TOKUZAWA, Naoki TAMURA, \\ Yoshio NAGAYAMA and LHD Experiment Group \\ National Institute for Fusion Science, 322-6 Oroshi-cho, Toki 509-5292, Japan \\ 1) Research Institute for Applied Mechanics, Kyushu University, Kasuga 816-8580, Japan
}

(Received 30 November 2015 / Accepted 14 March 2016)

\begin{abstract}
We have proposed and developed a digital correlation ECE (DCECE) measurement method using a highspeed sampling digitizer. This is the first report of the validation of the DCECE using actual data of plasma. The experiment was conducted in the 18th cycle experiments in the large helical device (2014 fiscal year) and a several $\mathrm{kHz}$ electron temperature fluctuation was detected in the high ion temperature plasma. The results were compared to the conventional radiometer type ECE measurement. Moreover, it was found that the correlation profile depends on the analytical parameters of the IF conversion.
\end{abstract}

(c) 2016 The Japan Society of Plasma Science and Nuclear Fusion Research

Keywords: ECE, direct sampling technique, correlation ECE, mesoscale phenomena, LHD

DOI: $10.1585 / \mathrm{pfr} .11 .2402072$

\section{Introduction}

Measuring the electron cyclotron emission (ECE) is a popular diagnostic tool in high magnetic field plasma devices. Because microwave diagnostics such as interferometry, reflectometry, and ECE are free from target plasma disturbance, they are expected to catch microphenomena including turbulence. In particular, because ECE can easily identify the measured plasma volume, ECE imaging has also been developed and installed on tokamaks. There is another approach to detect several millimeter-sized and several hundred $\mathrm{kHz}$ microphenomena. However, the sensitivity in the high-frequency region is low because ECE is derived from blackbody radiation. The correlation ECE (cECE) technique [1] is used to improve the sensitivity limit. In general, the spatial decorrelation technique is easy to use in existing measurement devices because the optics, including lens and mirrors, are the same as in existing devices. For the spatial decorrelation, the spatial resolution is an important parameter to obtain clear results of target phenomena. The radial spatial resolution is determined by the bandwidth of the bandpass filters in the intermediate frequency (IF); that is, the resolution cannot be changed easily.

Recently, the technology of analog-digital converter (ADC) has made remarkable advances. A digitizer with sampling rate more than several dozen $\mathrm{GHz}$ has been developed commercially. Because the frequency band of the downconverted IF wave is in the $\mathrm{GHz}$ range, we can obtain the IF waveform digitally. Typically, digitizers have been used to develop components in the telecommunications in-

author'se-mail: tsuchiya.hayato@lhd.nifs.ac.jp

*) This article is based on the presentation at the 25th International Toki Conference (ITC25). dustry.

We have proposed the digital correlation ECE (DCECE) measurement, which is based on the correlation ECE and the IF band direct sampling technique [2]. Applying the direct sampling technique to plasma experiments enables the flexible spatial resolution ECE measurement. In this study, we report on the observation of the electrostatic fluctuation by DCECE and compare these observations with the results of the conventional filter bank radiometer ECE.

\section{Analysis Procedure of DCECE}

In the case of the large helical device (LHD), whose maximum magnetic field is approximately $3 \mathrm{~T}$, the frequency of the second harmonic ECE is $100-150 \mathrm{GHz}$ in the maximum field. After passing the high/low pass filter, the RF:ECE is downconverted to the lower side band (LSB)/upper side band (USB) intermediate frequency (IF) of up to $20 \mathrm{GHz}$ by mixing with a proper coherent local oscillator (LO). In a conventional filter bank radiometer system, the generated IF is selected by the bandpass filter [3]. In the case of the filter bank system in LHD, the band width is $1 \mathrm{GHz}$, which corresponds to $2 \mathrm{~cm}$ spatial resolution in the radial direction. The radiometers are detected by the power of the selected IF and the power can be reconstructed to the electron temperature. The fluctuation of the power is obtained by ADC as the electron temperature fluctuation. The typical sampling rate is $100 \mathrm{kHz}$ to $1 \mathrm{MHz}$.

The direct sampling technique of IF is used in the ECE measurement instead of the analog filter bank system. The IF waveform is directly obtained by the fast ADC whose 


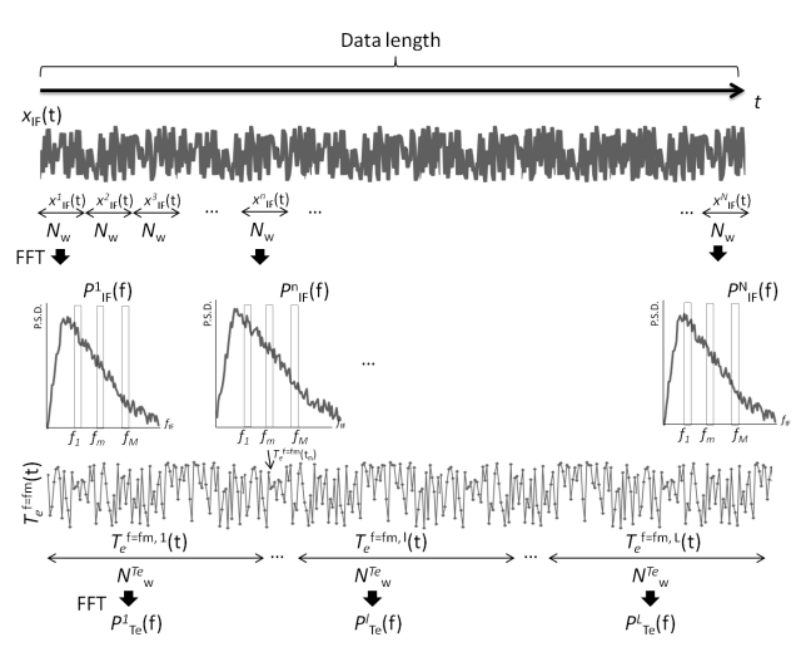

Fig. 1 DCECE data analysis procedure. The IF spectra can be calculated from each data window $x_{\mathrm{IF}}^{\mathrm{n}}(\mathrm{t})$ with length $N_{\mathrm{w}}$. The electron temperature fluctuation $T_{\mathrm{e}}^{\mathrm{f}=\mathrm{fm}}(\mathrm{t})$ is obtained as the fluctuation of the IF power spectrum. The spectrum of the electron temperature fluctuation $p_{\mathrm{Te}}^{1}(\mathrm{f})$ is also calculated from the data window $T_{\mathrm{e}}^{\mathrm{f}=\mathrm{fm}}(\mathrm{t})$ by the FFT. For correlation analysis, a large $N_{\text {spectra }}^{\mathrm{Te}}$ is desirable.

bandwidth is at least several GHz. We can reconstruct the electron temperature radial profile as the power spectra of the IF signal and obtain the electron temperature fluctuation as the fluctuation of the power spectrum of the IF signal. Figure 1 shows the DCECE data analysis procedure. The time series data of the IF waveform with sampling rate of $1 / \mathrm{d} t_{\mathrm{IF}}$ is divided to data windows with data length $N_{\mathrm{w}}$ that corresponds to the inverse of the sampling rate of the electron temperature fluctuation $\mathrm{d} t_{\mathrm{Te}}$ if smoothing procedure in the time domain is not applied. Each divided data is converted to complex components of the spectra by the fast Fourier transform, and the power spectrum of IF is calculated. The integrated power in the IF frequency window is proportional to the electron temperature. The width of each IF frequency window corresponds to the width of the bandpass filter in the conventional filter bank system; that is, the width of the bandpass filter determines the spatial resolution. In this manner, we can obtain multichannel electron temperature fluctuation data like the data of a conventional filter bank system. The coherence is also calculated by the same CECE procedure. More than one independent spectrum $N_{\text {spectra }}^{\mathrm{Te}}$ is necessary to calculate the coherence. Therefore, the data length of the IF waveform is an important parameter. As aforementioned, we can select several parameters of the correlation analysis, although the sampling rate and data length of the IF waveform are fixed by the specifications of the ADC. Because the memory volume in the ADC is limited, the data length is defined by setting the sampling rate of IF. If it is assumed that each channel of ADC has $512 \mathrm{MB}$, the data length that can be obtained from one shot corresponds to $6.4 \mathrm{~ms}$ under the condition of $80 \mathrm{GHz}$ sampling. The relation of the typical parameters of ADC and DCECE analysis is summarized in
Table 1 Typical parameters of the ADC and DCECE analysis.

\begin{tabular}{c|c|c} 
& $\begin{array}{c}\text { For } \\
\text { high-frequency } \\
\text { phenomena }\end{array}$ & $\begin{array}{c}\text { For } \\
\text { low-frequency } \\
\text { phenomena }\end{array}$ \\
\hline $\begin{array}{c}\text { Sampling rate } \\
\text { of IF 1/d } t_{\mathrm{IF}}\end{array}$ & $80 \mathrm{GHz}$ & $10 \mathrm{GHz}$ \\
\hline $\begin{array}{c}\text { Data length } \\
\text { (time) }\end{array}$ & $6 \mathrm{~ms}$ & $51 \mathrm{~ms}$ \\
\hline $\begin{array}{c}\text { Virtual } \\
\text { sampling rate } \\
\left(1 / \mathrm{d} t_{\mathrm{Te}}\right)\end{array}$ & $4.8 \mathrm{MHz}$ & $0.6 \mathrm{MHz}$ \\
\hline $\begin{array}{c}\text { Window } \\
\text { length for the } \\
\text { FFT of the } T_{\mathrm{e}} \\
\text { waveform }\end{array}$ & $\begin{array}{c}\left(2^{14} \mathrm{points}\right) \\
\begin{array}{c}\text { Number of } \\
\text { spectra } N_{\text {spectra }}^{\text {Te }}\end{array}\end{array}$ & $\begin{array}{c}13.4 \mathrm{~ms} \\
\left(2^{13} \mathrm{points}\right)\end{array}$ \\
\hline
\end{tabular}

Table 1. Before the plasma experiment, we must determine the sampling rate (i.e., data length) for the time scale of the target phenomena in plasmas. In this study, to compare the results of the well-known several $\mathrm{kHz}$ MHD fluctuation measured by the conventional filter bank system ECE, the sampling rate of IF is set at $10 \mathrm{GHz}$. Because the typical frequency of target phenomena is several $\mathrm{kHz}$, the virtual sampling rate of the electron temperature time series data is set at $600 \mathrm{kHz}$. Because the width of each IF frequency window is a free parameter, we analyzed the DCECE results by changing the width of the IF frequency window, i.e., the radial spatial resolution.

\section{Results}

In LHD, the electron temperature fluctuation derived from the MHD instability is successfully detected by DCECE using a LabMaster 10-36Zi (Teledyne Lecroy Co.). The typical ADC specifications are shown in Table 2 (see details in ref. [4]). The discharge is a high ion and electron temperature-oriented plasma, which is heated by the neutral beam injection (NBI) and electron cyclotron heating $(\mathrm{ECH})$. The electron temperature of the startup phase at $t \sim 3-3.7 \mathrm{~s}$ is anomalously high because of the effect of the nonthermal electron generated by ECH. The high ion temperature plasma is obtained at $t \sim 4.7 \mathrm{~s}$ by NBI. Because the data length of DCECE is not sufficiently long to obtain the time evolution of the discharge, the start trigger of DCECE is set at $t=4.7 \mathrm{~s}$, when the radial pressure gradient is steep and the instability is easily excited. By using the conventional filter bank system, it is confirmed that the electrostatic fluctuation whose frequency is approximately $4-5 \mathrm{kHz}$ is excited at $\rho \sim 0.5$ while the stored energy is relatively high, as shown in Fig. 2.

Because the sampling rate is $10 \mathrm{GHz}$-sampling to detect several kilo Hertz fluctuations, the Nyquist frequency 
Table 2 Typical specifications of the LabMaster 10-36Zi [4].

\begin{tabular}{|l|l|}
\hline Sample Rate & $80 \mathrm{GS} / \mathrm{s}$ \\
\hline Bandwidth & $36 \mathrm{GHz}$ \\
\hline Intersegment Time & $1 \mu \mathrm{s}$ \\
\hline Vertical resolution & $\begin{array}{l}8 \mathrm{bits} \text {; up to 11 bits with } \\
\text { enhanced resolution } \\
\text { (ERES) }\end{array}$ \\
\hline Maximum Acquisition & $512 \mathrm{Mpts} / \mathrm{Ch}$ \\
\hline
\end{tabular}

(a)

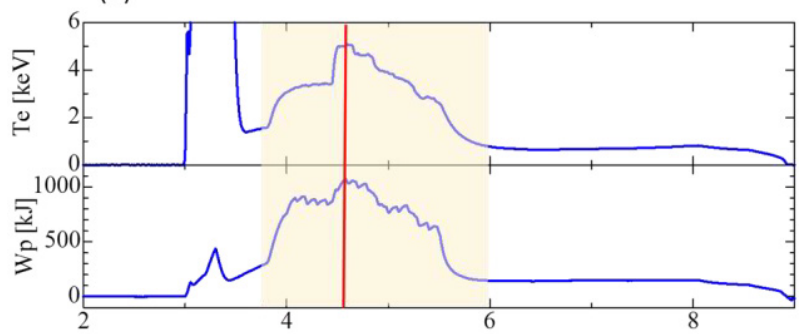

(b)

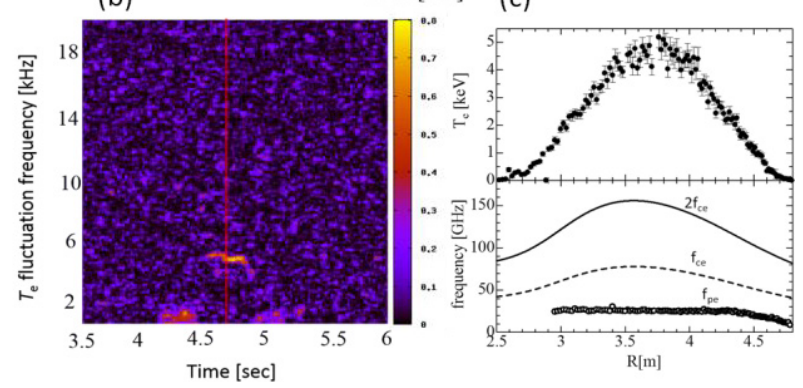

Fig. 2 (a) Electron temperature $\left(T_{\mathrm{e}}\right)$ and stored energy $\left(W_{\mathrm{p}}\right)$. (b) Coherence of electron temperature fluctuation measured by conventional ECE. The coherence is calculated from the data of the adjacent channel. The measured radial position is approximately $4.1 \mathrm{~m}$. The hatched area in (a) corresponds to the time range in (b). The vertical line at $t$ $=4.7 \mathrm{~s}$ corresponds to the time slice in Fig. 3. (c) Radial profile of the electron temperature and the profiles of the electron plasma frequency $\left(f_{\text {pe }}\right)$, electron cyclotron frequency $\left(f_{\mathrm{ce}}\right)$, and 2 nd harmonics of the electron cyclotron frequency $\left(2 f_{\text {ce }}\right) .2 f_{\text {ce }}$ is used in the ECE measurement.

is $5 \mathrm{GHz}$. The mixer, which is used for making the IF signal, has the frequency characteristic that 0.5 to $18 \mathrm{GHz}$ is effective that is, the effective band frequency for DCECE is $0.5-5 \mathrm{GHz}$. In our analysis procedure, the $0.5-4.5 \mathrm{GHz}$ IF is the effective band for avoiding an alias.

In the DCECE analysis procedure, the IF band between $0.5 \mathrm{GHz}$ and $4.5 \mathrm{GHz}$ can be digitally divided into any number of channels $N_{\mathrm{ch}}$. Figure 3 shows the comparison between the results of the conventional filter bank system ECE and DCECE. The coherence of each channel is calculated using the correlation with the fluctuation of the spatially adjacent channel. The comparison of the number of divided channels in the DCECE analysis is also described. It is found that the $4 \mathrm{kHz}$ fluctuation can be de-

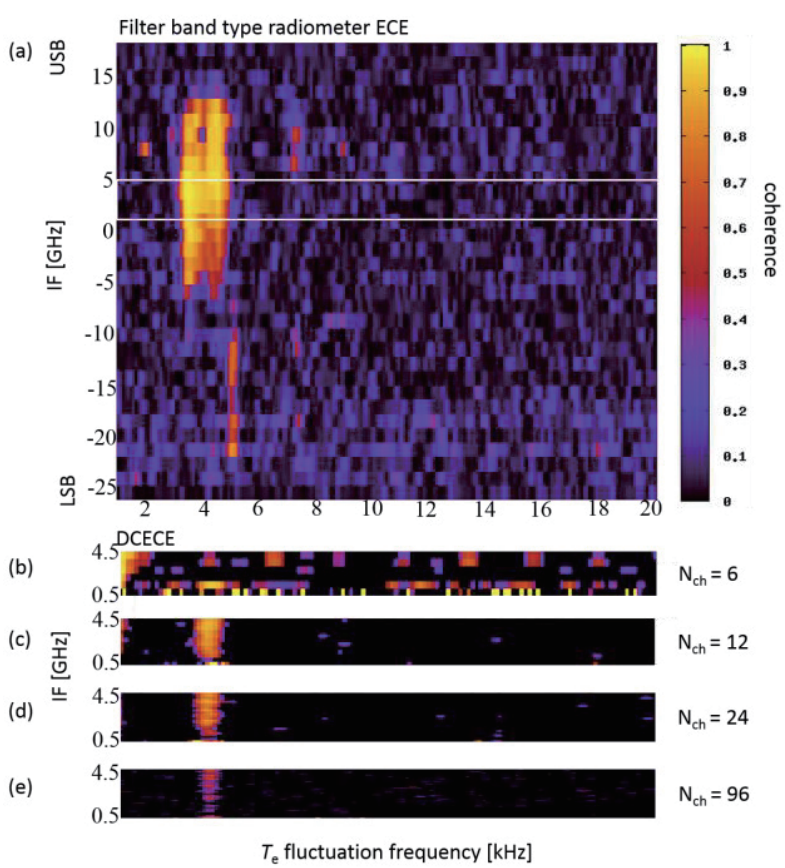

Fig. 3 (a) Coherence of the electron fluctuation obtained by the conventional filter bank system ECE at $t=4.7 \mathrm{~s}$ in Fig. 2 . The data of USB and LSB are shown together. The vertical IF axis can be reconstructed to the radial position. The $1 \mathrm{GHz}$ of IF corresponds to the $2 \mathrm{~cm}$ resolution. (b-e) Coherence of the electron fluctuation obtained by DCECE. The IF band is $0.5-4.5 \mathrm{GHz}$, which corresponds to the rectangular frame in Fig. 3 (a). The number of virtual channel $N_{\text {ch }}$ is (b) 6, (c) 12 , (d) 24, and (e) 96, respectively.

tected by DCECE for $N_{\mathrm{ch}}=12,24$, and 96. For $N_{\mathrm{ch}}=$ 6 , the fluctuation cannot be detected and this is attributed to the strong effect of smoothing. Conversely, for $N_{\mathrm{ch}}=$ 96 , the coherence is relatively lower than that for $N_{\mathrm{ch}}=$ 12 or 24 . Large $N_{\text {ch }}$ means making a narrow IF band channel. The signal level of the narrow band channel decreases, which is observed in the case of the analog band pass filter. Moreover, it is considered that the decreasing signal level in DCECE has a similar effect. It is also observed that the fluctuations are more sharply detected than the conventional filter bank system ECE.

Another example of detecting fluctuations higher than $10 \mathrm{kHz}$ is shown in Fig. 4. In general, the high-frequency fluctuation in the ECE measurements deteriorates because the ECE is regarded as blackbody radiation [3]. In the DCECE analysis procedure, the sensitivity limit is improved by using the simple ensemble procedure. In Fig. 4, the DCECE can detect small size phenomena around IF approximately in the range of $3.5-4 \mathrm{GHz}$. Because the IF resolution of conventional ECE is $1 \mathrm{GHz}$, the fluctuation cannot be exposed. The resolution of the IF band in DCECE is high enough to measure such small phenomena. However, we must keep in mind that the signal level of the high resolution is not occasionally sufficient. 


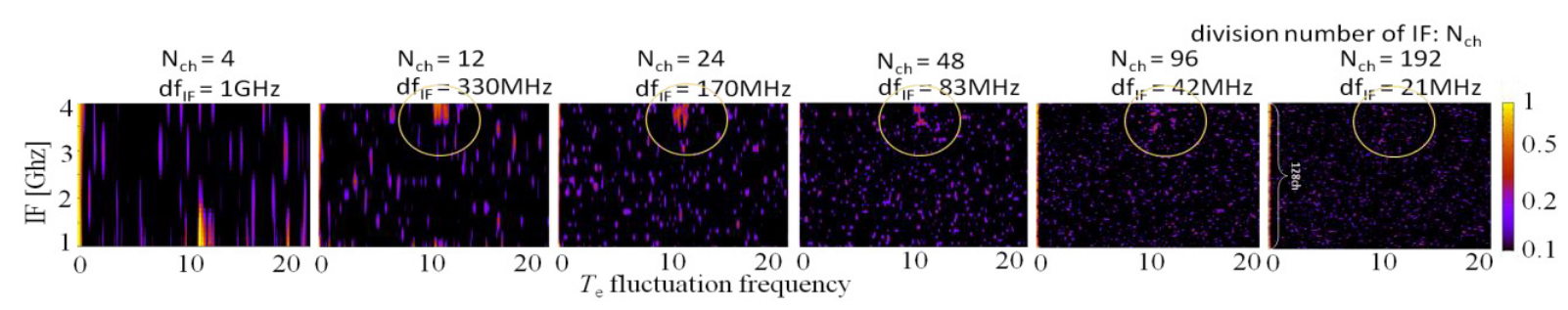

Fig. 4 Coherence map obtained by DCECE. Each figure is calculated from the same data. The coherence in the area is marked by a circle and is relatively high for $N_{\mathrm{ch}}=12,24,48$, and 96 .

As we have observed, the MHD fluctuations can be detected if the data length is sufficiently long and there is a possibility to detect small size phenomena that are smaller than the spatial resolution of the conventional filter bank system ECE.

\section{Summary}

The electrostatic fluctuation in the high-temperature plasma of the LHD can be observed by both conventional filter bank radiometer ECE measurements and the proposed DCECE. This is the first example of the electrostatic mode observation by DCECE. It is confirmed that several $\mathrm{kHz}$ fluctuations with strong correlation are more sharply detected than with the conventional filter bank system ECE. The spatial resolution can be varied arbitrarily; however, there are appropriate parameters to the target phenomena. For observing fluctuations higher than $20 \mathrm{kHz}$, the DCECE will be used in turbulence experiments.

The proposed DCECE can detect the fluctuations in the high-temperature plasma of LHD, which is also observed using the conventional filter bank radiometer ECE measurements. This is the first example of the MHD mode observation by the DCECE. It is confirmed that several $\mathrm{kHz}$ fluctuations with strong correlation are more sharply detected than the conventional filter bank system ECE. This suggests that the resolution of the IF spectra in DCECE is better than the width of the bandpass filters, even if smoothing is applied to the IF spectra frequency domain. The spatial resolution can be varied, but there are appropriate parameters to the target phenomena. In the future, the task is to observe fluctuations higher than $20 \mathrm{kHz}$. DCECE will be applied to a turbulence experiment.

\section{Acknowledgements}

This work was supported by the budget of the National Institute for Fusion Science and was done in collaboration with the Research Institute for Applied Mechanics, Kyushu University. The authors would like to thank Teledyne Leroy Japan Corporation for technical support.

[1] C. Watts, Fusion Sci. Technol. 52, 176 (2007).

[2] H. Tsuchiya et al., Plasma Fusion Res. 9, 3402021 (2014).

[3] H. Tsuchiya et al., Plasma Fusion Res. 6, 2402114 (2011).

[4] http://teledynelecroy.com/ 